\title{
Homozygous mutation in Atlastin GTPase 1 causes recessive hereditary spastic paraplegia
}

\author{
Lena Willkomm ${ }^{1,2}$, Raul Heredia ${ }^{3}$, Katrin Hoffmann ${ }^{4}$, Haicui Wang ${ }^{1,2}$, Thomas Voit ${ }^{5}$, Eric P Hoffman ${ }^{3}$ \\ and Sebahattin Cirak ${ }^{1,2,3,6}$
}

\begin{abstract}
Hereditary spastic paraplegia (HSP) is an extremely heterogeneous disease caused by mutations of numerous genes leading to lower limb spasticity (pure forms) that can be accompanied by neurological symptoms (complex forms). Despite recent advances, many causal mutations in patients remain unknown. We identified a consanguineous family with the early-onset HSP.

Whole-exome sequencing revealed homozygosity for a novel Atlastin GTPase 1 gene stop mutation in three affected siblings.

Heterozygous parents and siblings were unaffected. This was unexpected as mutations in the Atlastin 1 gene are known to cause autosomal dominant HSP. But our study showed that Atlastin 1 mutations may cause autosomal recessively inherited paraplegia with an underlying loss-of-function mechanism. Hence, patients with recessive forms of HSP should also be tested for the
\end{abstract} Atlastin 1 gene.

Journal of Human Genetics (2016) 61, 571-573; doi:10.1038/jhg.2016.6; published online 18 February 2016

Hereditary spastic paraplegias (HSPs) or spastic paraplegias (SPGs) are genetically highly heterogeneous disorders that occur in 4-10 out of 100000 individuals. ${ }^{1}$ HSPs are caused by mutations of genes encoding for proteins involved in the maintenance of corticospinal tract neurons. ${ }^{2}$ They primarily lead to progressive axonal degradation and lower limb spasticity. HSPs can be classified into pure (uncomplicated) HSPs or complex (complicated) HSPs if associated with additional symptoms such as intellectual disability, seizures, ataxia, peripheral neuropathy, skin abnormalities and visual defects. Mutations in numerous genes have been found to cause HSP (73 spastic gait disease loci and 55 SPG genes)., ${ }^{3,4}$ All four different modes of inheritance are known-autosomal dominant (AD; 12 genes); autosomal recessive (AR; 41 genes); X-linked (3 genes) and mitochondrial (1 gene). So far, only the SPG72 (REEP2) and SPG4 (SPAST) gene have been reported to be inherited autosomal dominantly and recessively. ${ }^{5,6}$

To provide genetic diagnosis despite the genetic heterogeneity whole-exome sequencing (WES) was performed as described previously ${ }^{7}$ and variant filtering was performed according to rare AR inheritance. Further experimental details are shown in Supplementary Materials and Methods.

In this consanguineous Arabic family, healthy parents who along with unaffected children did not show any neurological symptoms presented three out of six siblings suffering from HSP. The oldest affected sibling (subject 3) was born with a birth weight of $3.5 \mathrm{~kg}$ after normal pregnancy. The neonatal period was unremarkable but later motor milestone delay occurred with sitting at 8 months and walking at 15 months. First symptoms of spasticity were noticed with increased toe walking and increased muscle tone of the hip adductors. Due to the progressive spasticity, an operative release of several tendons was performed at 8 years to enable standing. Speech development was slow and learning difficulties were suggested but his IQ was never tested. On examination at 15 years, the occipitofrontal circumference was $54 \mathrm{~cm}$ (25th centile). He could take a few steps without help and had severe spasticity in the lower limbs (Figure 1a) with fixed flexion contractures of the hips and knees $\left(15^{\circ}\right)$, and very limited ankle movement. The patellar tendon, biceps and triceps reflexes were exaggerated. The Babinski sign was positive bilaterally. Movements of hands and diadochokinesis were slow, but he showed good, although slow, fine motor skills. We noted a reduction of distal muscle force down to MRC 4/5, which also affected the hands including atrophy of intrinsic hand muscles and swan-neck positioning on extension. The vibration sense was reduced in lower limbs but pain and touch senses were normal. Nerve conduction velocities of the motor N. peronaeus and sensory $\mathrm{N}$. suralis could not be determined as no potentials were detected during measurement. He reported problems with bladder control. At his latest clinical follow-up at 18 years, he was able to stand and walk with crutches.

The second affected male sibling (subject 6) was born after normal pregnancy and development in the early life was normal. Spasticity was detected at 18 months of age and independent walking was only achieved with 2.5 years. He also showed signs of speech and cognitive

\footnotetext{
${ }^{1}$ Institute of Human Genetics, University Hospital Cologne, Cologne, Germany; ${ }^{2}$ Center for Molecular Medicine Cologne (CMMC), University of Cologne, Cologne, Germany; ${ }^{3}$ Research Center for Genetic Medicine, Children's National Medical Center, Washington, DC, USA; ${ }^{4}$ Institute of Human Genetics, Martin-Luther-University Halle-Wittenberg, Halle an der Saale, Germany; ${ }^{5}$ Institute of Myology, Pierre and Marie Curie University, UPMC-INSERM UMR 974, Paris, France and ${ }^{6}$ Department of Peadiatrics, University Hospital Cologne, Cologne, Germany

Correspondence: Dr S Cirak, Institute of Human Genetics, University Hospital Cologne, Gebäude 47 (Frauenklinik), 9. OG, Kerpener Straße 34, 50931 Cologne, Germany. E-mail: Sebahattin.Cirak@uk-koeln.de

Received 1 April 2015; revised 9 January 2016; accepted 15 January 2016; published online 18 February 2016
} 
a

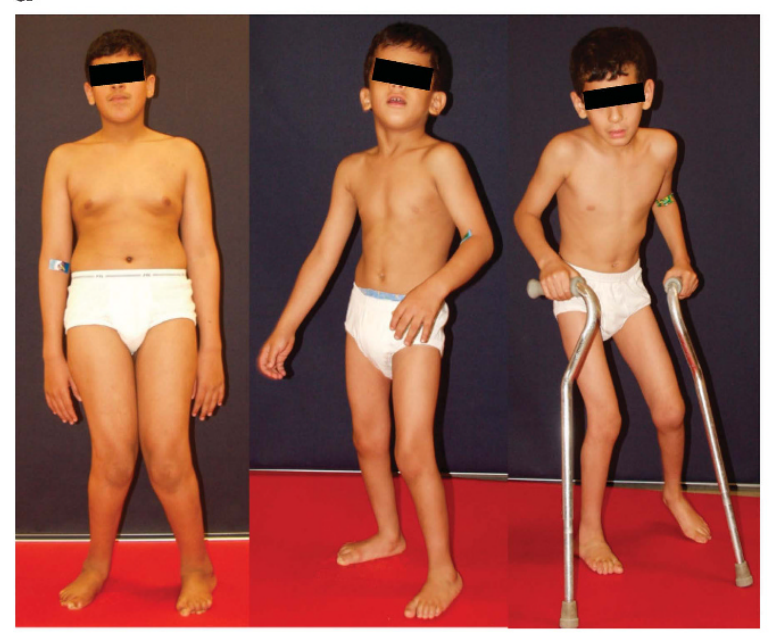

C

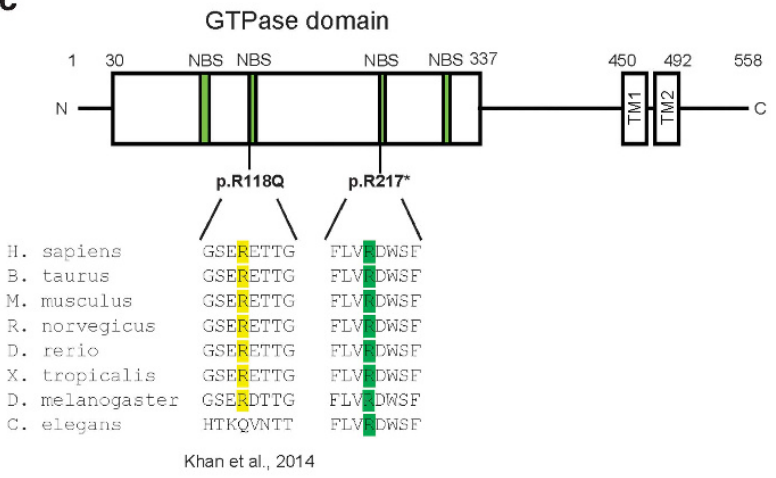

b

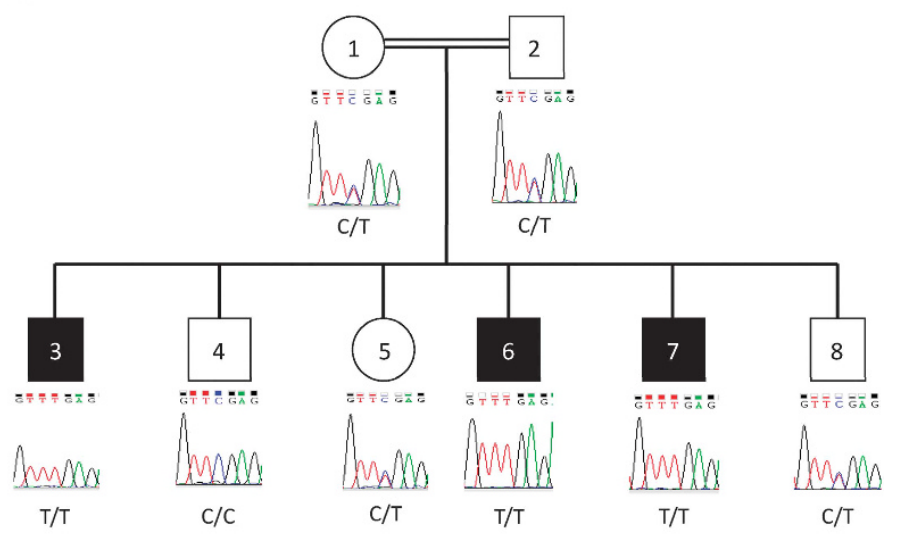

d

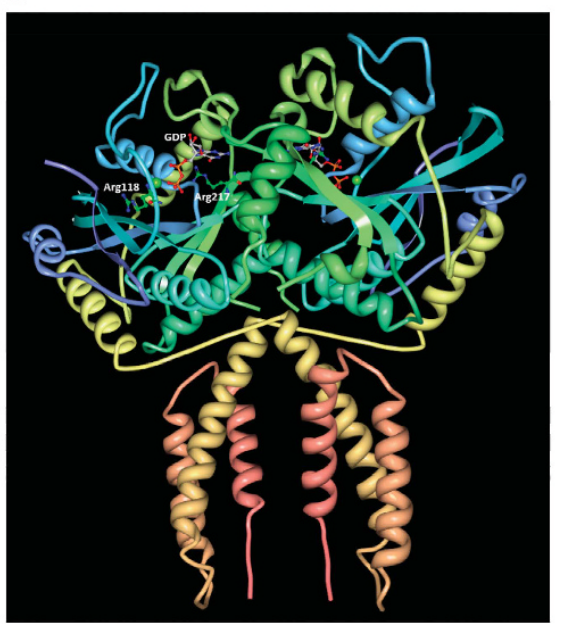

Figure 1 (a) Affected males at the age of 15 years (subject 3), 7 years (subject 6) and 6 years (subject 7). Clinical images clearly show the spasticity predominantly in the legs. The patients did not have ptosis. (b) Pedigree including the sequence chromatograms of the family showing the autosomal recessive inheritance. Consanguineous parents are heterozygous whereas the all affected offspring's carry the homozygous stop mutation $c .649 \mathrm{C}>T$ (p.R217*). The healthy siblings are either heterozygous or carrying the wild-type allele. (c) Relative position of the described recessive mutations in the ATL1 gene. (d) ATL1 (3QNU) crystal structure of the cytosolic domain of human Atlastin 1 in complex with GDP, hexagonal form. The three-dimensional modeling showing the mutation sites. The mutation reported here as well as the c.353G >A (p.R118Q) mutation described by Khan et al. are located within a nucleotide-binding site (NBS) of the GTPase domain. Both mutations lie in absolutely conserved residues.

developmental delay. Due to progressive spasticity, he underwent surgery at the age of 5 years for hip release and hamstring lengthening. During the clinical examination at the age of 7 years, he did not show any signs of spasticity yet in the upper limbs, but in the lower limbs he showed exaggerated reflexes and a positive Babinski sign. His gait was spastic with tendency to toe walking. The third male sibling (subject 7) presented a similar clinical picture at the age of 5 years with lower limb spasticity and a positive Babinski sign. He also had learning difficulties and slow speech. From the clinical history, he achieved walking with 2 years of age and had surgery at the age of 3 years for Achilles tendon and hamstring release. The heterozygous carrier parents, currently aged 40 years, had no neurological symptoms on examination.

WES revealed a homozygous stop mutation in the Atlastin GTPase 1 (ATL1) at c.649C>T, p.R217* (NM_015915) in two affected siblings and Sanger sequencing confirmed co-segregation of the mutation within the entire family (Figure 1b). ATL1 mutations usually cause AD SPG3A or Hereditary Sensory Neuropathy Type 1D and account for about $10 \%$ of all AD HSPs. This type is known as type
SPG3A and the most frequent cause of HSP with an onset before the age of 10 years. ${ }^{8}$ The Atlastin 1 protein is a dynamin GTPase superfamily member and predominantly present in the central nervous system, particularly within corticospinal neurons where it has been identified as a crucial protein in maintaining endoplasmic reticulum, Golgi morphology and vesicle trafficking. ${ }^{9}$

SPG3A is generally triggered by missense mutations with a dominant-negative effect on the tetrameric GTPase domain and a gain-of-function mechanism has been proposed $^{10}$ with varying phenotypes depending on mutation location, environmental and genetic factors. ${ }^{11}$ However, a few reports show recessive or complicated inheritance. Varga et al. ${ }^{12}$ reported a family with complicated inheritance. They describe one family with three siblings carrying homozygous mutation, and a fourth sibling showing a HSP phenotype with a heterozygous genotype. This could be due to modifier gene variants within the family. The recessive mutation described by Khan et al. ${ }^{13}$ and the one described here indicate a pathogenesis due to loss-of-function. The homozygous stop mutation p.R217* likely leads to nonsense-mediated decay of mRNA or instable protein and 
function of the tetrameric ATL1 GTPase complex in healthy heterozygous carriers, which does not interfere with the wild-type allele.

Khan et al. ${ }^{13}$ reported a consanguineous Pakistani family in which six males presented homozygous missense variant in the ATL1 (c.353G > A, p.R118Q; NM_001127713). Phenotypically, patients showed pure SPG with an onset before 2 years of age. Clinical features included spastic gait, toe walking, hyperreflexia of the lower limbs, pes cavus, reduced vibration sense, peripheral numbness and tingling, urinary bladder hyperactivity and scoliosis. Cognitive function was normal. ${ }^{13}$

All three of our patients had learning difficulties, so far only one patient with dominant ATL1 mutation has been described to have mental retardation. ${ }^{14}$ It remains to be shown whether other patients with recessive ATL1 mutations might have features of complex HSP, as Atlastin 1 is also expressed in other cells than motor neurons. This finding is also supported by a functional brain imaging study in SPG3A-linked HSP patients that revealed a relative decrease in glucose metabolism in the frontal cortex (where ATL1 is mainly expressed) associated with frontal cognitive impairment. ${ }^{15,16}$ Both mutations lie within nucleotide-binding sites of the well-conserved GTPase domain of ATL1 (Figures 1c,d). Possibly, mutation-induced reduction or even total loss-of-function might be causing the phenotype. Our hypothesis is that in many of the earlier published dominant ATL1 mutations the pathomechanism is dominant negative as ATL1 forms a dimer. ${ }^{17}$

This would be in line with AR inheritance as it has been suggested previously for HSP caused by several other genes. ${ }^{18}$

In conclusion, the finding of a novel $\mathrm{AR}$ trait adds to the understanding of the inheritance patterns and understanding of SPG3A. We suggest that patients with unsolved forms of HSP should be tested for mutations in SPG3A to provide genetic diagnosis and enhance our understanding of ATL1-related HSP.

\section{CONFLICT OF INTEREST}

The authors declare no conflict of interest.

\section{ACKNOWLEDGEMENTS}

This work was supported by Muscular Dystrophy Association, USA, and Deutsche Forschungsgemeinschaft, Germany, grants to Dr SC.
1 Erichsen, A. K., Koht, J., Stray-Pedersen, A., Abdelnoor, M. \& Tallaksen, C. M. E. Prevalence of hereditary ataxia and spastic paraplegia in southeast Norway: a population-based study. Brain 132, 1577-1588 (2009).

2 Harding, A. E. Classification of the hereditary ataxias and paraplegias. Lancet $\mathbf{3 2 1}$ 1151-1155 (1983).

3 Finsterer, J., Löscher, W., Quasthoff, S., Wanschitz, J., Auer-Grumbach, M. \& Stevanin, G. Hereditary spastic paraplegias with autosomal dominant, recessive, X-linked, or maternal trait of inheritance. J. Neurol. Sci. 318, 1-18 (2012).

4 Lo Giudice, T., Lombardi, F., Santorelli, F. M., Kawarai, T. \& Orlacchio, A. Hereditary spastic paraplegia: clinical-genetic characteristics and evolving molecular mechanisms. Exp. Neurol. 261, 518-539 (2014).

5 Esteves, T., Durr, A., Mundwiller, E., Loureiro, J. L., Boutry, M., Gonzalez, M. A. et al. Loss of association of REEP2 with membranes leads to hereditary spastic paraplegia. Am. J. Hum. Genet. 94, 268-277 (2014).

6 Lindsey, J. C., Lusher, M. E., McDermott, C. J., White, K. D., Reid, E., Rubinsztein, D. C. et al. Mutation analysis of the spastin gene (SPG4) in patients with hereditary spastic paraparesis. J. Med. Genet. 37, 759-765 (2000).

7 Punetha, J., Monges, S., Franchi, M. E., Hoffman, E. P., Cirak, S. \& Tesi-Rocha, C. Exome sequencing identifies DYNC1 $\mathrm{H} 1$ variant associated with vertebral abnormality and spinal muscular atrophy with lower extremity predominance. Pediatr. Neurol. 52 , 239-244 (2014).

8 Namekawa, M., Ribai, P., Nelson, I., Forlani, S., Fellmann, F., Goizet, C. et al. SPG3A is the most frequent cause of hereditary spastic paraplegia with onset before age 10 years. Neurology 66, 112-114 (2006).

9 Byrnes, L. J. \& Sondermann, H. Structural basis for the nucleotide-dependent dimerization of the large G protein atlastin-1/SPG3A. Proc. Natl Acad. Sci. USA 108, 2216-2221 (2011).

10 Alvarez, V., Sanchez-Ferrero, E., Beetz, C., Diaz, M., Alonso, B., Corao, A. I. et al. Mutational spectrum of the SPG4 (SPAST) and SPG3A (ATL1) genes in Spanish patients with hereditary spastic paraplegia. BMC Neurol. 10, 89 (2010).

11 Magariello, A., Tortorella, C., Citrigno, L., Patitucci, A., Tortelli, R., Mazzei, R. et al. The p.Arg416Cys mutation in SPG3a gene associated with a pure form of spastic paraplegia. Muscle Nerve 45, 919-920 (2012).

12 Varga, R. E., Schule, R., Fadel, H., Valenzuela, I., Speziani, F., Gonzalez, M. et al. Do not trust the pedigree: reduced and sex-dependent penetrance at a novel mutation hotspot in ATL1 blurs autosomal dominant inheritance of spastic paraplegia. Hum. Mutat. 34, 860-863 (2013).

13 Khan, T. N., Klar, J., Tariq, M., Anjum Baig, S., Malik, N. A., Yousaf, R. et al. Evidence for autosomal recessive inheritance in SPG3A caused by homozygosity for a novel ATL1 missense mutation. Eur. J. Hum. Genet. 22, 1180-1184 (2014).

14 Orlacchio, A., Montieri, P., Babalini, C., Gaudiello, F., Bernardi, G. \& Kawarai, T. Lateonset hereditary spastic paraplegia with thin corpus callosum caused by a new SPG3A mutation. J. Neurol. 258, 1361-1363 (2011).

15 Zhao, X. P., Alvarado, D., Rainier, S., Lemons, R., Hedera, P., Weber, C. H. et al. Mutations in a newly identified GTPase gene cause autosomal dominant hereditary spastic paraplegia. Nat. Genet. 29, 326-331 (2001).

16 Terada, T., Kono, S., Ouchi, Y., Yoshida, K., Hamaya, Y., Kanaoka, S. et al. SPG3A-linked hereditary spastic paraplegia associated with cerebral glucose hypometabolism. Ann. Nucl. Med. 27, 303-308 (2013).

17 Bian, X., Klemm, R. W., Liu, T. Y., Zhang, M., Sun, S., Sui, X. W. et al. Structures of the atlastin GTPase provide insight into homotypic fusion of endoplasmic reticulum membranes. Proc. Natl Acad. Sci. USA 108, 3976-3981 (2011).

18 Novarino, G., Fenstermaker, A. G., Zaki, M. S., Hofree, M., Silhavy, J. L., Heiberg, A. D. et al. Exome sequencing links corticospinal motor neuron disease to common neurodegenerative disorders. Science 343, 506-511 (2014).

Supplementary Information accompanies the paper on Journal of Human Genetics website (http://www.nature.com/jhg) 